\title{
Weakly nonlinear oscillations of gas column driven by self-sustained sources
}

\author{
Viktor Hruška ${ }^{1, *}$, Michal Bednařík ${ }^{1}$, and Milan Červenka ${ }^{1}$ \\ ${ }^{1}$ Czech Technical University in Prague, Faculty of Electrical Engineering, Technická 2, 16627 Prague 6, Czech Republic
}

\begin{abstract}
Self-sustained sources coupled to some sort of resonator have drawn attention recently as a subject of nonlinear dynamics with many practical applications as well as interesting mathematical problems from the chaos theory and the theory of synchronizations. In order to mimic the self-sustainability arising from physical background the van der Pol equation is commonly used as a model (e.g. vortex induced noise, flowstructure interactions, vocal folds motion etc.). In many cases the sound field inside the resonator is strong enough for weakly nonlinear formulation based on the Kuznetsov model equation to be employed. An array of sources governed by the inhomogeneous van der Pol equation coupled to the nonlinear acoustic wave equation is studied. The one dimensional constant cross-section open resonator with zero radiation impedance is assumed. The focus is on the main features such as mode-locking, harmonics generation and build-up from infinitesimal fluctuations.
\end{abstract}

\section{Introduction}

Many complex phenomena from the fields of aeroacoustics and fluid-structure interactions involve self-sustained sound sources [1-5]. It occures quite often that the sources are situated within a waveguide with a strong sound field which they drive and receive feedback at the same time (e.g. the sound generated by the airflow through a corrugated pipe). The main concern of the following study is to propose a framework to deal with such scenarios phenomenologically, i.e. without computationally very demanding direct numerical simulations based on the compressible Navier-Stokes equations.

We expect that the self-suistained source should be able to start oscillations only from omnipresent infinitesimal fluctuations and further that there should be some sort of saturation mechanism so the oscillations do not grow infintely (note that this saturation should be a feature of the oscillator, not the acoustic medium). The both conditions are satisfied when the van der Pol oscillator is used.

From the perspective of nonlinear acoustics we shall remain within the weakly nonlinear formulation. There are multiple ways to describe the nonlinear standing waves inside an one-dimensional resonator and for this reason we employed the Kuznetsov model equation (KE) (see e.g. $[6,7])$.

Again, there are various mechanisms through which the acoustic field could interact with the sources. We choose the local acoustic velocity to be the quantity mediating the feedback. The reason for this choice is e.g. the Howe's formula (Howe's energy corollary) describing the feedback effect of the existing sound field on the generated vortex sound power (see e.g. [8]).

*e-mail: viktor.hruska@fel.cvut.cz

\section{Theory}

The wave equation in the common linear (d'Alembertian) form might be extended for the cases of finite amplitudes by introducing the nonlinear terms. We start with the onedimensional Kuznetsov equation expressed in terms of an acoustic velocity potential $\varphi$ (see e.g. [6]):

$$
c_{0}^{2} \frac{\partial^{2} \varphi}{\partial x^{2}}-\frac{\partial^{2} \varphi}{\partial t^{2}}=\frac{\partial}{\partial t}\left(\frac{\partial \varphi}{\partial x}\right)^{2}+(\gamma-1) \frac{\partial \varphi}{\partial t} \frac{\partial^{2} \varphi}{\partial x^{2}}-\zeta \frac{\partial^{3} \varphi}{\partial x^{2} \partial t}
$$

where $c_{0}, \gamma, \zeta$ denote the adiabatic sound speed, the adiabatic exponent and the diffusion coefficient respectively, $x$ is the spatial coordinate along the waveguide axis and $t$ is time. An integro-differential term describing the effects of thermoviscous boundary layer was ommited and the diffusion coefficient was increased instead (see e.g. [9] for further commentary).

Considering the velocity potential formulation a source term corresponding to the (volume density of) force distribution $f$ which might be supplemented to Eq. (1) should take the form:

$$
\int \frac{\partial f}{\partial t} \mathrm{~d} x
$$

We assume that the source is represented by an array of point-like sources and therefore:

$$
f(x, t)=\sum_{m} g_{m}(t) \delta\left(x-x_{m}\right) .
$$

where $x_{m}$ is the location of the $m$-th source and $\delta(x)$ denotes the Dirac function and $g_{m}(t)$ is the instantaneous value of the $m$-th oscillating force term. The last expression (3) may be integrated according to (2) and substituted to (1) to obtain the the first of the model equations: 


$$
\begin{gathered}
c_{0}^{2} \frac{\partial^{2} \varphi}{\partial x^{2}}-\frac{\partial^{2} \varphi}{\partial t^{2}}= \\
\frac{\partial}{\partial t}\left(\frac{\partial \varphi}{\partial x}\right)^{2}+(\gamma-1) \frac{\partial \varphi}{\partial t} \frac{\partial^{2} \varphi}{\partial x^{2}}-\zeta \frac{\partial^{3} \varphi}{\partial x^{2} \partial t}-\sum_{m} \frac{\partial g_{m}}{\partial t} \mathrm{H}\left(x-x_{m}\right)
\end{gathered}
$$

where $\mathrm{H}$ denotes the Heaviside step function.

The acoustic velocity $v$ and the pressure $p$ are calculated from the velocity potential as

$$
\begin{aligned}
& v=\frac{\partial \varphi}{\partial x} \\
& p=-\rho_{0} \frac{\partial \varphi}{\partial t}+\frac{\rho_{0}}{2 c_{0}^{2}}\left(\frac{\partial \varphi}{\partial t}\right)^{2}-\frac{\rho_{0}}{2 c_{0}^{2}}+\sum_{m} g_{m} \mathrm{H}\left(x-x_{m}\right)
\end{aligned}
$$

where $\rho_{0}$ is the ambient density and a small term due to dissipation was omitted in the pressure equation.

In order to mimic the self-sustainability of the sources the governing equations for $g_{m}$ can take the van der Pol form. Hence of the proposed equations:

$$
\ddot{g}_{m}+\varepsilon \omega\left(\alpha^{2} g_{m}^{2}-1\right) \dot{g}_{m}+\omega^{2} g_{m}=\left.\omega \eta \frac{\partial \varphi}{\partial x}\right|_{x=x_{m}},
$$

where the dot denotes the derivative with respect to $t$ and $\varepsilon, \omega, \eta$ denote a coefficient of nonlinearity, an eigenfrequency and the feedback coefficient respectively. The parameter $\alpha$ governs the limit cycle size. It is straightforward to show that for an autonomous van der Pol oscillator the amplitude of oscillations is $2 \alpha^{-1}$ (see e.g. [10]).

Next we introduce dimensionless quantities

$$
\begin{gathered}
t=\frac{L}{\pi c_{0}} \tau, \quad x=L \sigma, \quad \varphi=\frac{L c_{0}}{\pi} \Phi \\
g=\rho_{0} c_{0}^{2} G, \quad p=\pi^{2} \rho_{0} c_{0}^{2} P
\end{gathered}
$$

and recast the governing equations as

$$
\begin{array}{r}
\frac{1}{\pi^{2}} \frac{\partial^{2} \Phi}{\partial \sigma^{2}}-\frac{\partial^{2} \Phi}{\partial \tau^{2}}=\frac{\partial}{\partial \tau}\left(\frac{\partial \Phi}{\partial \sigma}\right)^{2}+(\gamma-1) \frac{\partial \Phi}{\partial \tau} \frac{\partial^{2} \Phi}{\partial \sigma^{2}}- \\
-\zeta^{*} \frac{\partial^{3} \Phi}{\partial \sigma^{2} \partial \tau}+\frac{1}{\pi^{2}} \sum_{m} \frac{\partial G_{m}}{\partial \tau} \mathrm{H}\left(\sigma-\sigma_{m}\right) \\
\ddot{G}_{m}+\varepsilon v\left(\beta^{2} G_{m}^{2}-1\right) \dot{G}_{m}+v^{2} G_{m}=\left.v \eta^{*} \frac{\partial \Phi}{\partial \sigma}\right|_{\sigma=\sigma_{m}}
\end{array}
$$

where $v=\omega L / \pi c_{0}$ is the normalized frequency, $\beta=\alpha \rho_{0} c_{0}^{2}$ and $\eta^{*}, \zeta^{*}$ denote the modified feedback coefficient and attenuation coefficients respectively and dot represents the time derivative with respect to $\tau$ now.

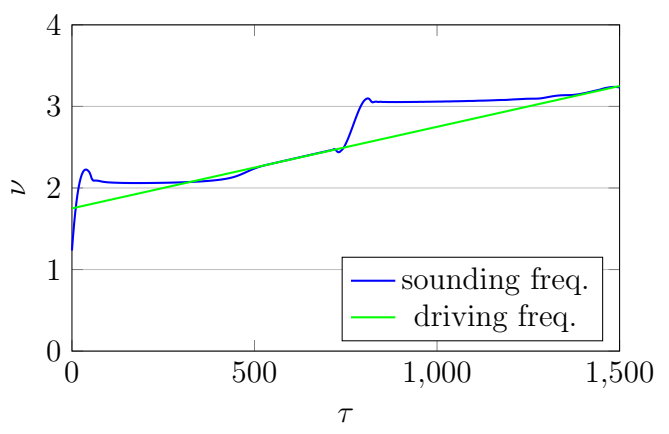

Figure 1. Instantaneous frequencies of the acoustic velocity at the open end obtained by the Hilbert transform (blue) and the driving frequency (green).

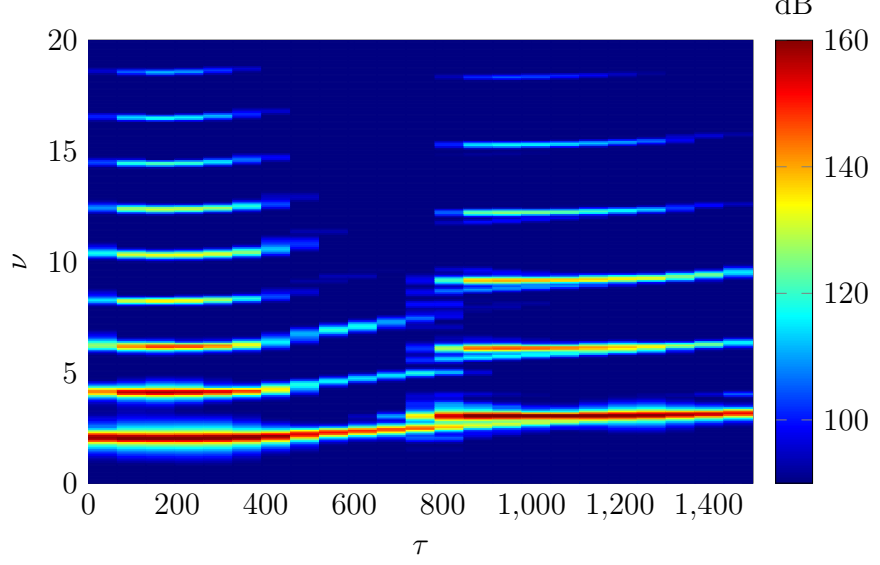

Figure 2. Spectrogram of the linear sweep between the 2nd and the 3rd resonant frequency. The input signal is the acoustic velocity at the open end. The depicted values is the sound velocity level with the reference value $5 \cdot 10^{-8} \mathrm{~m} / \mathrm{s}$.

\section{Numerical experiment}

Range of possible scenarios for numerical simulations is very wide. Hence we limit ourselves to a convenient example of the main features. A narrow open resonator is investigated assuming that the ends are pressure release surfaces and hence $p=0$ there. Equations (8-9) are solved by the 4th order central finite difference scheme in spatial domain in order to obtain a set of ordinary differential equations with respect to time. They are solved using the Python library SciPy [11] (scipy . integrate.odeint based on FORTRAN odepack [12]). The boundary conditions are introduced using ghost points.

In order to show the key phenomena the following setup was simulated. Five sources were placed equidistantly in the waveguide. Their frequencies were equal and slowly growing (a linear sweep), starting slightly below the 2nd pipe resonance and ranging towards the 3rd one. This feature could be motivated e.g. by the whistling of corrugated pipes where the driving frequency depends on the mean flow velocity through the pipe [13]. The other parameters were chosen as follows: $\eta^{*}=0.5, \zeta^{*}=5 \cdot 10^{-4}, \varepsilon=0.005$, $\beta=10^{4}$. 
First we see that the sounding frequency is not growing linearly. In Fig. 1 is depicted the instantaneous frequency at the pipe open end (obtained by the Hilbert transformation). Clearly the nonlinear synchronization (modelocking) occurs near the resonance frequency although not matching it perfectly.

In the mode-locked states it is possible to build-up a strong sound field due to the resonance. The number of harmonics grows (see Fig. 2) but the shocks are not formed because of the above mentioned detuning. When the driving moves further out of resonance the high harmonics are attenuated. When the driving gets close enough to the next resonance a sudden sounding frequency shift takes place.

\section{Discussion}

The sources located at exact infinitesimal points are only a simple mathematical approximation of real sources. In a more realistic scenario the action of the force distribution would be limited to a small, yet finite part of the waveguide. We can replace the expression (3) by a more general $f(x, t)=g(t) S(x)$. Next we can rely on the fact that the sources for $\varphi$ should be step-like in space and therefore $\int S \mathrm{~d} x$ could be a well suited sigmoid function. Obviously a gaussian shape would be suitable for $S(x)$ but then we would have to deal with the error function. Instead the logistic curve might be employed

$$
\begin{aligned}
\int S(x) \mathrm{d} x & =C \frac{1}{1+e^{-s x}}, \\
S(x) & =C \frac{\sigma e^{-s x}}{\left(e^{-s x}+1\right)^{2}},
\end{aligned}
$$

where $s$ governs the slope of the curve and $C$ should be used for normalization.

The van der Pol oscillators generally have an oddharmonic spectrum. It is most likely that this feature is responsible for breaking a textbook case of successive harmonics build-up towards the shock formation. Since the sources are not perfectly sinuous the harmonics inside the resonator grow by pairs: the "proper successive even" and the "stronger receptive odd". Nevertheless, this trait is due to the van der Pol equation. It does not reflect any particular physical phenomenon and can be partly controlled by the nonlinearity parameter $\varepsilon$ in Eq. (9).

Although the aeroacoustic sources are the main motivation convective effects on the sound field have not been taken into account. This is valid approximation as far as the flow is uniform and of low Mach number. Small corrections could be introduced by the Prandtl-Glauert transform [8].

\section{Summary}

We have seen the main features of the Kuznetsov equation driven by the array of the van der Pol-like sources (modelocking, sudden oscillation regime change, generation of higher harmonics). Such systems are common in the field of aeroacoustics of turbulent media and beyond. Similar set-up involving oscillations in liquid metal was studied by Makarov et al. [14].

The future research should deal with better understanding of the governing parameters, cases in which the shocks take place and effects of the radiation and convective losses at the open ends.

This work was supported by the GACR grant No. 18-24954S and by the Grant Agency of the Czech Technical University in Prague, grant No. SGS17/130/OHK3/2T/13.

\section{References}

[1] M. Facchinetti, E. de Langre, F. Biolley, Journal of Fluids and Structures 19, 123 (2004)

[2] M.L. Facchinetti, E. de Langre, F. Biolley, European Journal of Mechanics - B/Fluids 23, 199 (2004)

[3] M.L. Facchinetti, E. de Langre, F. Biolley, C. R. Mecanique 330, 451 (2002)

[4] A. Tondl, R. Nabergoj, Chaos, Solitons \& Fractals 4, 2193 (1994)

[5] L. Mathelin, E. de Langre, European Journal of Mechanics - B/Fluids 24, 478 (2005)

[6] M. Bednarik, M. Cervenka, The Journal of the Acoustical Society of America 135, EL134 (2014)

[7] M.F. Hamilton, D.T. Blackstock, et al., Nonlinear Acoustics (Acoustical Society of America, Melville, NY, 2008)

[8] A. Hirschberg, S.W. Rienstra (2004), free online source, http://www.win.tue.nl/ sjoerdr/ papers/les-swr-mh.pdf

[9] Y.A. Ilinskii, B. Lipkens, T.S. Lucas, T.W.V. Doren, E.A. Zabolotskaya, The Journal of the Acoustical Society of America 104, 2664 (1998)

[10] P. Maneville, Instabilities, Chaos and Turbulence (Imperial College Press, Londýn, 2010)

[11] E. Jones, T. Oliphant, P. Peterson et al., SciPy: Open source scientific tools for Python (2001-), [Online; accessed <today>], http://www. scipy.org/

[12] A.C. Hindmarsh, IMACS Transactions on Scientific Computation 1, 55 (1983)

[13] V. Debut, J. Antunes, M. Moreira, A Phenomenological Model for Sound Generation in Corrugated Pipes., in Proceedings of ISMA (2007)

[14] S. Makarov, R. Ludwig, D. Apelian, The Journal of the Acoustical Society of America 105, 2216 (1999) 